\title{
Evidence-Based Tailored Nutrition Educational Intervention Improves Adherence to Dietary Guidelines, Anthropometric Measures and Serum Metabolic Biomarkers In Early-Stage Breast Cancer Patients: A Prospective Interventional Study.
}

Ilaria Trestini

University of Verona

Isabella Sperduti

Biostatistics Unit, IRCCS Regina Elena National Cancer Institute

Alberto Caldart

University of Verona

Clelia Bonaiuto

University of Verona

Elena Fiorio

University of Verona

Veronica Parolin

University of Verona

Valentina Zambonin

University of Verona

Sara Zanelli

University of Verona

Daniela Tregnago

University of Verona

Alice Avancini

University of Verona

Marco Cintoni

Università di Roma Tor Vergata

Sara Pilotto

University of Verona

Maria Cristina Mele

Universitario Agostino Gemelli IRCCS, Università Cattolica del Sacro Cuore

Antonio Gasbarrini

Università Cattolica Del Sacro Cuore 


\section{Giovanni Scambia}

Policlinico Universitario Agostino Gemelli IRCCS

\section{Michele Milella}

University of Verona

\section{Giampaolo Tortora}

Universitario Agostino Gemelli IRCCS, Università Cattolica Del Sacro Cuore

\section{Emilio Bria ( $\nabla$ emilio.bria@unicatt.it )}

Universita Cattolica del Sacro Cuore https://orcid.org/0000-0002-2333-704X

\section{Luisa Carbognin}

University of Verona

\section{Research Article}

Keywords: Breast cancer, Nutritional intervention, Dietary guidelines, Weight loss, Body mass index change, Serum metabolic biomarkers change.

Posted Date: February 12th, 2021

DOI: https://doi.org/10.21203/rs.3.rs-220551/v1

License: (c) (i) This work is licensed under a Creative Commons Attribution 4.0 International License. Read Full License

Version of Record: A version of this preprint was published at The Breast on December 1st, 2021. See the published version at https://doi.org/10.1016/j.breast.2021.08.008. 


\section{Abstract}

Purpose: The impact of the adherence to dietary guidelines of early-stage breast cancer (EBC) patients on body composition changes during treatment is not entirely defined. This study aimed to evaluate the role of an evidence-based nutrition educational intervention, according to adherence to dietary guidelines, in EBC patients.

Methods: This prospective study included EBC patients, candidates for neoadjuvant/adjuvant therapy. Patients received an evidence-based tailored nutrition educational intervention. The adherence to dietary guidelines, anthropometric and dietary assessments, and blood glucose and lipid profile tests were evaluated at baseline and after a 12-months nutritional intervention.

Results: Two hundred and forty-three patients were enrolled (median age 49 years). At baseline, 38.3\% and $23.9 \%$ of patients were overweight and obese, weight gain $\geq 5 \%$ and central obesity were observed in $47.3 \%$ and $52.7 \%$ of patients, respectively, and adherence to dietary guidelines was low (median Med-Diet score: 6). After 12-months of nutritional intervention (median follow-up: 22 months), adherence to dietary guidelines significantly increased (median Med-Diet score: $12, p<0.0001$ ). High adherence to dietary guidelines (defines as Med-Diet score $\geq 10)$ significantly correlated with: 1$)$ weight loss $\geq 5 \%(21.8 \%$ vs. $2.5 \%, p=0.003$ ); 2) median BMI drop (from $25.6 \mathrm{~kg} / \mathrm{m}^{2}$ to $24.4 \mathrm{~kg} / \mathrm{m}^{2}, p=0.003$ ); 3) lower prevalence of central obesity $(38.2 \%$ vs. $7.2 \%, p=0.01) ; 4)$ improvement in blood glucose levels and lipid profile.

Conclusion: This study suggests that an evidence-based tailored nutrition educational intervention during treatment for EBC significantly increases overall adherence to dietary guidelines, and it improves both anthropometric measures and serum metabolic biomarkers in patients with high adherence.

\section{Implications For Practice}

Despite the consistent evidence linking excess adiposity to early-stage breast cancer (EBC) prognosis, the implementation and assessment of adherence to lifestyle interventions have been limited to date. The present study suggests that high adherence to dietary guidelines, achieved by an early evidence-based tailored nutrition educational intervention, correlates with an improvement in anthropometric measures and serum metabolic profile in patients undergoing treatment for EBC. Thus, EBC patients should receive a nutritional assessment immediately after diagnosis, and strategies, including dietary intervention, should be incorporated into clinical practice to control the body weight and, consequently, potentially enhance the disease outcome.

\section{Introduction}

Overweight and obesity are highly prevalent in patients affected by early-stage breast cancer (EBC) [1]. Most women experienced weight gain from diagnosis, during, and after treatment for breast cancer [2]. Particularly, chemotherapy-related side effects may negatively impact on the patients' diet, limiting the enjoyment of eating and leading to changes in dietary pattern. The reduction of physical activity, the 
ovarian failure for premenopausal women, and the decrease in basal metabolic rate, which promote an impairment in energy balance as well as an influence on their nutritional status, may significantly worsen the anthropometric parameters [3]. Concerning survival outcome, several observational studies demonstrate that both baseline overweight and weight gain after diagnosis, during adjuvant and/or neoadjuvant therapy, seem to negatively affect the prognosis of EBC patients [4-6]. Particularly, the excess of adiposity may detrimentally affect the quality of life and self-esteem and it may increase the risk of breast cancer recurrence, second primary malignancies, [7] glucose and lipid dysregulation and, consequently, comorbidities, such as type 2 diabetes and cardiovascular disease [8] in both pre- and postmenopausal women [9]. All these implications are particularly relevant for EBC patients receiving (any) systemic therapy, who usually experience variations in serum metabolic biomarkers regardless of the presence of serious comorbidities at diagnosis [10]. Contextually, EBC patients who are underweight are suggested to have a poorer prognosis compared to normal weight and to increase the risk of complications due to malnutrition, such as bone fracture risk $[4,11]$.

Recent studies underline valuable benefits of a healthy diet, implementing a low-fat dietary pattern associated with increased vegetables, fruit, and whole-grain intake, as well as physical activity and weight control for women affected by EBC [12-14]. A high level of adherence to the Mediterranean-style dietary pattern was shown to improve overall health in EBC survivors by decreasing their risk of nonbreast cancer-related mortality $[15,16]$. . Nevertheless, the nutritional assessment and the impact of the adherence to dietary guidelines, in terms of body size changes, during the treatment phase for EBC patients are not entirely defined in clinical practice [17]. Indeed, the interventional studies commonly recruit patients 3 to 5 years post-diagnosis [18] and their success depends on the patients' adherence to the intervention [19]. Given these perspectives, this prospective study aimed to evaluate the impact of an evidence-based nutritional intervention on adherence to dietary guidelines and to explore its effectiveness in terms of body weight and serum metabolic biomarkers change, according to adherence to dietary guidelines, in patients affected by EBC undergoing systemic treatment.

\section{Materials And Methods}

\section{Study design and patient population}

This prospective study enrolled EBC patients, diagnosed and addressed to receive neoadjuvant/adjuvant chemotherapy and/or hormonotherapy at the University of Verona, Azienda Ospedaliera Universitaria Integrata of Verona. Inclusion criteria were: 1 ) women > 18 years old; 2) diagnosis of operable and locally advanced (stage I-III) breast cancer scheduled for initiating neoadjuvant/adjuvant chemotherapy and/or hormonotherapy. Eligible patients were recruited by the medical staff at the first oncological visit. After recruitment, patients received a nutritional evaluation before starting the systemic treatment. Afterward, patients were submitted to an evidence-based tailored educational intervention by registered dietitians with documented skills for an evidence-dietetic practice in cancer patient care. Patients' baseline clinical characteristics, pathological tumor characteristics at diagnosis, and neoadjuvant/adjuvant treatment information were collected by medical staff. 
The study was approved by the local Ethics Committee and patient consent was obtained (Prot. 778CESC).

\section{Objective}

This study was designed to evaluate the variation in body weight, body mass index (BMI), waist circumference (WC), serum metabolic biomarkers, and emotional function as a consequence of a 12months evidence-based nutritional intervention, according to adherence to dietary guidelines.

\section{Dietary assessment}

Trained dietitians collected information about food consumption using three non-consecutive 24-hour dietary recall (24HR), in a face-to-face interview with the participants, including one weekend day and two weekdays, in order to better reflect the eating habits of the patients. At baseline and after 12 months, participants completed a validated questionnaire to assess adherence to the Mediterranean dietary guidelines [15]

The Mediterranean diet assessment tool (Med-Diet) consisted of 14 questions. Each question was scored with zero or one point. One point was given for using olive oil as the main fat for cooking, preferring white meat to red meat and daily consumption of four or more tablespoons of olive oil (including oil used for frying, dressing salads, etc.), two or more servings of vegetables, three or more pieces of fruit, less than one serving of red meat or sausage, less than one serving of animal fat and less than one cup of carbonated or sugary drinks. One point was also given for weekly intake of seven or more glasses of wine, three or more servings of legumes, three or more servings of fish, two shop-bought pastries or fewer, three or more servings of nuts, and two or more helpings of sofrito (a traditional sauce made with tomato, garlic, onion or leeks and sautéed with olive oil). Responses were added for a total value between 0 and 14 , with scores score ${ }^{3} 10$ indicating high adherence to Mediterranean dietary guidelines. Notably, although wine is an abundant source of polyphenols that have antioxidant functions, given its unfavorable role on breast cancer [7], alcohol intake may not be appropriate to include as a dietary goal for EBC. Thus, we excluded alcohol from the score calculation in the present analysis.

\section{Dietary intervention}

The dietary intervention was designed to improve adherence to dietary guidelines and to pursue and maintain healthy body weight and WC. The dietary intervention included an evidence-based tailored nutritional counseling by a trained dietitian and monitoring, through a monthly nutritional visit during and after treatments, involving individualized sample meal plans and recipe suggestions that were adjusted on personalized eating patterns and preferences (full details reported in the online supplementary materials).

\section{Sample Size and Statistical analysis}


The sample size was assessed assuming the maximum possible variability. In order to determine an adherence to dietary guidelines of $50 \%$ (hypothesis of maximum variability) after 12 -months of nutritional intervention, 243 patients were required, with a maximum Standard Error of 3\% (accuracy level $6 \%$ ). Patient's nutritional characteristics were tabulated and compared according to menopausal status at the time of EBC diagnosis since evidence suggested that premenopausal status may be a strong predictor of weight change [20]. Descriptive data are presented as median (with range or interquartile range [IQR]) for continuous variables, frequencies and percentages are reported for categorical variables. For continuous variables, comparisons among groups were performed with Mann-Whitney or KruskalWallis test. Pearson's chi-square ( $\chi 2)$ test and Fisher's exact test are used to assess differences in categorical variables. Analysis for paired data was carried with Wilcoxon test or McNemar test, when appropriate.

A multivariate logistic regression analysis was adopted using the backward stepwise method, to identify independent predictive factors of weight loss $\geq 5 \%$, prevalence of central obesity, BMI change and serum metabolic markers (glycemic, total cholesterol, HDL and triglycerides levels) alterations after 12 months. The included variables in the univariate analysis were age, menopausal status, treatment, axillary lymph nodes status and adherence to dietary guidelines. Odds ratios (ORs) with $95 \%$ confidence intervals ( $95 \%$ $\mathrm{Cl}$ ) were estimated for each variable in the context of univariate and multivariate analysis.

The level of significance was set at $p \leq 0.05$. SPSS software (SPSS version 20.0, SPSS Inc., Chicago, Illinois, USA) was used for all statistical evaluations.

\section{Results}

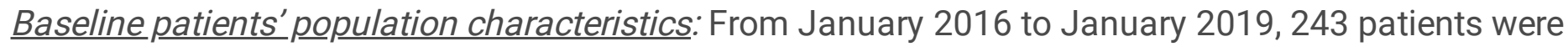
enrolled (Table 1). Median follow-up was 22 months (range 12-45). The median age at diagnosis was 49 years (range 27-83) and $99.2 \%$ of patients had an ECOG Performance Status 0. One hundred and thirtyone $(53.9 \%)$ patients were premenopausal at diagnosis. Patients were addressed to receive chemotherapy in neoadjuvant $(27.6 \%)$ or adjuvant (48.6\%) setting, with adjuvant (83.1\%) hormonotherapy in case of hormone receptor positivity. We registered 5 cases of local relapses, 3 cases of regional relapses, 9 cases of distant metastasis, and 3 deaths.

Baseline patients' nutritional evaluation: Table 2 provides data for baseline nutritional features in the overall population and according to menopausal status. Regarding baseline anthropometric characteristics, the median BMI was $25.6 \mathrm{~kg} / \mathrm{m}^{2}$ (IQR 22.5-29.1). The number of patients with normal weight was $101(41.6 \%)$, while $5(2.1 \%)$ patients were underweight, 93 (38.2\%) were overweight, and 58 (23.9\%) were obese, of whom 37 (63.8\%) obese class I, 9 (15.5\%) obese class II and $12(20.7 \%)$ obese class III. Furthermore, weight gain $\geq 5 \%$ was observed in 115 (47.3\%) patients, especially, in 61 (53\%) premenopausal and 54 (47\%) post-menopausal women. Central obesity, according to WC, was found in 128 $(52.7 \%)$ women, of whom $59(44 \%)$ in pre-menopausal versus $69(63.3 \%)$ in postmenopausal $(p=0.01)$. Notably, central obesity was significantly correlated with tumor size in the whole population $(37.5 \%$ for 
pT1, $58.8 \%$ for $\mathrm{pT} 2$, and $100 \%$ for $\mathrm{pT} 3-4, p=0.003$ ). Likewise, patients with central obesity were more likely to be affected by HER2-positive tumors ( $57.4 \%$ vs. $42.5 \%, p=0.03$ ). Many patients reported relevant nutritional impact symptoms: dyspepsia and constipation were present in 125 patients (51.4\%) and 152 patients (62.6\%), respectively. These symptoms were more prevalent in overweight and obese patients, as well as in patients who gained $\geq 5 \%$ of weight (Supplementary Tables 1 and 2 ). The median reported caloric intake was $1793 \mathrm{kcal} / \mathrm{die}$ (IQR 1469-2190) and median dietary fat intake was 35.3\% (IQR 29.240.9) of energy intake with $11.1 \%$ (IQR 7.7-13.1) of calories from saturated fat, while the median intake of dietary fiber was $17.2 \mathrm{~g}$ /day (IQR 12.4-23.1), as reported in Supplementary Table 3. Of note, there was a significant correlation between increased dietary fat intake and dyspepsia $(p=0.005)$. Concerning the association between blood tests and anthropometric measures (BMI and central obesity), patients with excess body weight and central obesity reported higher glycemic, total cholesterol, and triglycerides levels as well as lower HDL levels (Supplementary Tables 4 and 5). According to IPAQ short form scoring classification, $79 \%$ of patients resulted inactive, whereas $21 \%$ were minimally active. A significant correlation between physical activity levels and $\mathrm{BMI}$ (percentage of patients minimally active: $0 \%$ for $\mathrm{BMl}<18.5 \mathrm{~kg} / \mathrm{m}^{2}, 87.4 \%$ for $18.5 \leq \mathrm{BMI} \leq 24.9 \mathrm{~kg} / \mathrm{m}^{2}, 21.5 \%$ for $25 \leq \mathrm{BMl} \leq 29.9 \mathrm{~kg} / \mathrm{m}^{2}, 6.9 \%$ for $\mathrm{BMI} \geq 30$ $\left.\mathrm{kg} / \mathrm{m}^{2}, p=0.002\right)$, WC $<88 \mathrm{~cm}$ vs. $\geq 88 \mathrm{~cm}(38.3 \%$ vs. $15.6 \%$, respectively, $p=0.007)$ and weight gain $\geq 5 \%$ vs. $<5 \%(20.9 \%$ vs. $31.3 \%$, respectively, $p=0.02)$ was found.

Adherence to dietary guidelines: A low baseline adherence to dietary guidelines (median Med-Diet score: 6) was measured (Supplementary Table 6). Regarding the dietary intake patterns, only 79 (32.5\%) of women reported consuming 2 or more servings of vegetables a day, $71(29.2 \%) 3$ or more servings of fruit a day and $17(7 \%)$ patients expressed eating 3 or more servings of legumes a week as well as $16(6.6 \%)$ consuming 3 or more servings of fish or shellfish week. Besides, 109 (44.9\%) patients reported limiting consumption of sweet or carbonated beverages to less than one time a day. After the 12-months tailored evidence-based nutritional intervention, the adherence to dietary guidelines significantly increased compared to baseline (median Med-Diet score: 6 vs.12, p<0.0001). At 12 months, $51.4 \%$ of the intervention group reported consuming 2 or more servings of vegetables a day, which was significantly higher than the $32.5 \%$ adherence at baseline $(p<0.0001)$. The consumption of 3 or more servings of fruit a day increased from $29.2 \%$ (at baseline) to $58.1 \%$ at 12 months $(p<0.0001)$. High adherence to dietary guidelines (defines as a Med-Diet score $\geq 10$ ), was found in $152(62.6 \%)$ patients. No difference in terms of menopausal status, tumor characteristics, and treatments (chemotherapy with/without hormonotherapy vs. hormonotherapy alone) between patients with high and low adherence was reported. High adherence to dietary guidelines was significantly correlated with a weight loss $\geq 5 \%$ from the baseline $(21.8 \%$ vs. $2.5 \%, p=0.003)$ and a WC reduction $(37.4 \%$ vs. $11.9 \%, p=0.001)$. In this subset of patients, as a consequence of weight loss, there was a statistically significant drop in median BMI from $25.6 \mathrm{~kg} / \mathrm{m}^{2}$ to $24.4 \mathrm{~kg} / \mathrm{m}^{2}$ after the dietary intervention $(p=0.003)$. Consequently, the proportions of overweight and obese patients declined considerably and there was a significant reduction of patients with central obesity (Figure 1 [Panel A-C]). Furthermore, none of the patients classified as underweight were found after the nutritional intervention. 
Regarding blood tests, high adherence to dietary guidelines was associated with an improvement in blood glucose levels and plasma lipid profile, with lower cholesterol and triglycerides, and higher HDL cholesterol compared to baseline values (Figure 2 [Panel A]). No BMI, WC and circulating biological values' differences were reported for patients with low adherence to dietary guidelines (Figure 1 [Panel BD] and Figure 2 [Panel B]).

Predictive factors for weight loss $\geq 5 \%$, prevalence of central obesity, BMI category change, and serum metabolic markers alteration after 12 months: At multivariate analysis only the type of treatment and age were associated with weight loss $\geq 5 \%$ (OR 2.26, 95\% Cl 1.04-4.89, $p=0.039$ for chemotherapy vs chemotherapy+ hormonotherapy vs hormonotherapy) and prevalence of central obesity (OR 10.1, 95\% Cl $1.96-51.8, p=0.006$ for no vs yes), respectively. The menopausal status and the adherence to dietary guidelines were independent predictors of BMI change, as reported in Table 3. None of the factors has been shown to be associated with serum metabolic markers alteration (data not shown).

Quality of life assessment: Emotional function, according to the EORTC questionnaire, was evaluable for 211 out of 243 patients (86.8\%). Several patients experienced 'quite a bit' (G3) or 'very much' (G4) tension $(41.2 \%)$, worry (40.8\%), irritability (36\%), and depression (19\%). Associations between emotional function and anthropometric measures (baseline BMI and weight gain) are reported in Figures 3 and 4 . No difference in terms of tension, worry, irritability, and depression was found between patients with high and low baseline adherence to dietary guidelines. Of interest, overall, after the educational intervention, the WC reduction was correlated with lower rates of 'G3-G4' tension ( $11.7 \%$ vs. $8.6 \%, p=0.001)$, worry $(14.5 \%$ vs. $8.2 \%, p=0.001)$ and irritability $(13.2 \%$ vs. $8.8 \%, p=0.002)$.

\section{Discussion}

This prospective study suggests that an evidence-based tailored educational nutritional intervention during treatment for EBC can increase the adherence to dietary guidelines and improve the anthropometric measures and the serum metabolic biomarkers in patients with high adherence. Unlike other cancers, weight gain after EBC diagnosis represents a common and relevant issue, as reported by a series of observational studies, considering that women who are overweight or gain weight after diagnosis seem to be at greater risk for breast cancer recurrence and death compared with lighter women [20]. In these studies, the weight gain, considered clinically relevant when it is more than $5 \%$ of prediagnosis weight, was assessed in the first years after diagnosis or after the completion of adjuvant treatments [21]. Conversely, in our analysis, almost half of the patients already gained more than $5 \%$ of their usual weight before starting neoadjuvant/adjuvant chemotherapy or adjuvant hormonotherapy. Moreover, this weight gain needs to be examined within the context that, at diagnosis, many patients in our study were overweight (38.2\%), obese (23.9\%), and central obese (52.7\%). Concerning menopausal status at diagnosis, we did not detect any difference in the extent of weight increase between premenopausal and postmenopausal patients, while we reported more central obesity in postmenopausal 
women, strengthening the hypothesis that the excess of adiposity may be a breast cancer risk factor only for postmenopausal women [22]. The weight gain in EBC may be related and influenced by a combination of phycological factors and behavior [23]. In this context, we observed a significant correlation between both baseline BMI and weight gain with tension, worry, irritability, and depression, as well as with the levels of physical activity, measured by IPAQ. In line with previous evidence [24], many women of our cohort (79\%) were inactive. A significant decline in levels of physical activity is very frequent after EBC diagnosis, leading to change in body composition, as well as compromise physiological and psychological functions, potentially worsening breast cancer outcomes $[25,26]$. Besides, a change in the role of food, in eating behavior and nutrition status is common among women after diagnosis. Indeed, our cohort presented a high-fat dietary pattern, exceeding the $20 \%$ of total energy, as recommended by current evidence $[12,27]$, and a low fiber intake, which were both found to have independent effects on serum estrogen levels $[28,29]$. Although some women tend to opt for a healthier diet after diagnosis, there are reports that they find comfort in consuming less healthy foods, using them as a reward for the difficult period of therapy [30]. With regard to food consumption, we observed a low consumption of fruits, vegetables, and legumes and increased intake of high energy-dense and nutritionally poor foods, in line with previous findings [31].

Promising evidence suggested that weight gain may be prevented in EBC patients with diet intervention, achieving weight maintenance or loss [32,33]. The educational component of our intervention was based on international recommendations, promoting the Med-Diet, not using group educational sessions [34]. Patients may be more comfortable with a program that can address their specific needs and concerns, as well as reinforce the survivor-specific benefits of improved lifestyle factors. Moreover, the scheduled visits were by face-to-face contact during treatment and not by mail or telephone recall, as reported in several studies [35,36], ensuring a more active role of the patients in dietary change and, thus, determining probably better adherence to dietary guidelines.

The Med-Diet has been shown to support weight loss and improve the inflammatory profile in cardiac and diabetic groups [37]. Given the tendency for EBC patients to gain body weight and risk for metabolic syndrome, the Med-Diet may have a crucial role, promoting weight loss and offering multiple benefits due to fiber and antioxidants content. [38]. Several studies investigated the relationship between dietary interventions and weight management in EBC survivors, providing support for the efficacy for achievement weight loss [33]. However, a small number of nutritional interventional analyses focused on a Mediterranean-like dietary pattern [39-41].

After the 12-months evidence-based tailored nutritional intervention, dietary habits were changed, improving in plant food intake, and the adherence to dietary guidelines significantly increased compared to baseline. Consist with these data, a recent randomized trial, including 153 overweight and obese EBC survivors, reported that patients who received a dietary intervention significantly increased adherence to Med-Diet guidelines compared to patients who received informational brochures $(+22.5 \%$ vs. $+2.7 \%$, $p<0.001$ ) [42]. In our study, a high adherence, achieved by $62.6 \%$ of patients, significantly impacted weight loss, leading to a median BMI and WC drop. Particularly, high adherence Med-Diet resulted as a 
significant predictive factor in the multivariate analysis for BMI change. The proportion of overweight and obese patients considerably declined (from $39.5 \%$ to 13.8 and from $9.2 \%$ to $3.3 \%$, respectively, $p=0.003$ ) and there was a significant reduction of patients with central obesity (from $38.2 \%$ to $7.2 \%, p=0.01$ ). Furthermore, after the dietary intervention, none of the patients were found underweight. This represents a relevant aspect since that a series of observational studies reported an association between low $\mathrm{BMI}$ and increased risk of local recurrence and death $[43,44]$. . Focus on the anthropometric measures' change, Finocchiaro et al. evaluated 100 women previously treated for EBC submitted to an individualized intervention program based on WCRF/AICR guidelines and modeled on Med-Diet. They observed a significant improvement of adherence to the Med-Diet, with a statistically significant decrease in mean $\mathrm{BMI}$ and WC. However, this analysis recruited patients when they completed any chemotherapy treatment [40], thus when most of the weight gain has already occurred.

Concerning the serum metabolic biomarkers, in our study, patients with high adherence to dietary guidelines after the educational intervention reported an improvement in fasting levels of blood glucose and lipid profile, providing insight into the biological mechanisms through nutritional profile may impact on EBC outcome, reduce comorbidities and improve overall health. Indeed, both neoadjuvant and adjuvant treatments appear to determine a significant increase of blood glucose levels and worsening of lipid profile $[10,45]$. These alterations, regardless of the specific cause, may persist up to 24 months after diagnosis and may be responsible for the worse prognosis in these patients [46]. On the other hand, focusing on patients with low adherence to dietary guidelines, no BMI, WC, and blood tests' differences were observed. Thus, our data emphasized the relevance of adherence to dietary guidelines in improving body weight and serum metabolic biomarkers. Considering that weight gain and altered metabolic profile after diagnosis has been frequently reported for EBC patients, especially among women receiving systemic chemotherapy [47], these data confirm the efficacy of dietary counselling by a registered dietitian in preventing weight and metabolic negative changes. Of note, overall, after the educational intervention, the WC reduction was correlated with an enhancement of emotional function, confirming previous evidence suggesting that improving weight-related distress plays an important role in increasing the well-being of EBC patients [48].

A limit of this study is represented by the fact that the body composition was evaluated by the WC ( surrogate measure of the visceral fat area), without the lean tissue mass assessment leading to potentially missed diagnoses of sarcopenic obesity [49]. Besides, the effect of the dietary intervention on the EBC recurrence and survival is not evaluated, due to the short median follow-up. However, available evidence suggests the prognostic impact of nutritional intervention $[12,19]$. In this regard, several trials evaluating the impact of weight loss and other lifestyle changes after breast cancer diagnosis on recurrence risk and mortality are currently ongoing $[50,51]$. Nonetheless, the present study presents several strengths, including an accurate evaluation of anthropometric measures and dietary intake detected by trained dietitians, not based on patient's self-reporting. Likewise, the nutritional intervention was customized on an individual basis, with regular visits directly with the patient and not by mail or telephone recall. Notably, we recruited women before starting the systemic therapy and we included 
patients regardless of their baseline BMI, evaluating the effect of the dietary intervention on body weight also in underweight patients.

\section{Conclusions}

This prospective study provides some insights as to the potential impact of a pragmatic, real-world dietary intervention in EBC patients during treatment. The results suggest that high adherence to dietary guidelines, achieved by an early evidence-based tailored educational nutritional intervention, correlates with an improvement in body weight, BMI, WC and serum metabolic profile in patients undergoing adjuvant or neoadjuvant treatment for EBC. Considering the potential negative prognostic impact of overweight, obesity, and weight gain for EBC patients, this study supports to adopt a nutritional assessment and an evidence-based tailored dietary intervention at diagnosis and during treatment in order to control the body weight, and, consequently, potentially enhance the disease outcome.

\section{Declarations}

\section{Declarations}

Not applicable.

\section{Acknowledgments}

I.T., C.B., G.T., E.B. and L.C. were supported by a grant of the Lega Italiana contro I Tumori (LILT-Bando di ricerca sanitaria 2016 - 5X1000 anno 2014). E.B. is currently supported by the Associazione Italiana per la Ricerca sul Cancro (AIRC) under Investigator Grant (IG) No. IG20583. GT is supported by AIRCwith IG No.18599, AIRC 5x1000 No. 21052. EB is currently supported by Institutional funds of Università Cattolica del Sacro Cuore (UCSC-project D1-2020). LC is supported by AIRC with My First AIRC Grant (MFAG) No. 25149.

\section{Compliance with Ethical Standards:}

\section{Conflicts of interest}

The authors declared the following potential conflicts of interest with respect to the research, authorship, and/or publication of this article: IT reported speakers' fees from Mylan and Fresenius Kabi., L.C. received honoraria or speakers' fee from Novartis, Istituto Gentili and Eli-Lilly. S.P. received honoraria or speakers' fee from Astra-Zeneca, Eli-Lilly, BMS, Boehringer Ingelheim, MSD andRoche. M.M. received speakers' honoraria from and participated on advisory boards for: EUSA Pharma, Pfizer, MSD, AstraZeneca, MerckSerono and Mylan. E.B. received speakers' and travels' fee from MSD, Astra-Zeneca, Celgene, Pfizer, Helsinn, Eli-Lilly, BMS, Novartis and Roche. E.B received consultant's fee from Roche, Pfizer. E.B. received institutional research grants from Astra-Zeneca, Roche. The other authors have no conflicts of interest to declare. 


\section{Ethical approval}

All procedures performed in studies involving human participants were in accordance with the ethical standards of the institutional and/or national research committee and with the 1964 Helsinki declaration and its later amendments or comparable ethical standards.

\section{Informed consent}

Informed consent was obtained from all individual participants included in the study.

\section{References}

1. Greenlee H, Shi Z, Sardo Molmenti CL et al. Trends in Obesity Prevalence in Adults With a History of Cancer: Results From the US National Health Interview Survey, 1997 to 2014. J Clin Oncol 2016; 34: 3133-3140.

2. Nyrop KA, Deal AM, Shachar SS et al. Weight trajectories in women receiving systemic adjuvant therapy for breast cancer. Breast Cancer Res Treat 2020; 179: 709-720.

3. Custodio ID, Marinho Eda C, Gontijo CA et al. Impact of Chemotherapy on Diet and Nutritional Status of Women with Breast Cancer: A Prospective Study. PLoS One 2016; 11: e0157113.

4. Nichols HB, Trentham-Dietz A, Egan KM et al. Body mass index before and after breast cancer diagnosis: associations with all-cause, breast cancer, and cardiovascular disease mortality. Cancer Epidemiol Biomarkers Prev 2009; 18: 1403-1409.

5. Kogawa T, Fujii T, Fouad TM et al. Impact of change in body mass index during neoadjuvant chemotherapy and survival among breast cancer subtypes. Breast Cancer Res Treat 2018; 171: 501 511.

6. Fang Q, Huang J, Gan L et al. Weight Gain during Neoadjuvant Chemotherapy is Associated with Worse Outcome among the Patients with Operable Breast Cancer. J Breast Cancer 2019; 22: 399-411.

7. World Cancer Research Fund/American Institute for Cancer Research. Continuous Update Project Expert Report 2018. Diet, nutrition, physical activity and breast cancer survivors. Available at dietandcancerreport.org. In.

8. Howe LR, Subbaramaiah K, Hudis CA, Dannenberg AJ. Molecular pathways: adipose inflammation as a mediator of obesity-associated cancer. Clin Cancer Res 2013; 19: 6074-6083.

9. Chan DS, Vieira AR, Aune D et al. Body mass index and survival in women with breast cancersystematic literature review and meta-analysis of 82 follow-up studies. Ann Oncol 2014; 25: 19011914.

10. Dieli-Conwright CM, Wong L, Waliany $S$ et al. An observational study to examine changes in metabolic syndrome components in patients with breast cancer receiving neoadjuvant or adjuvant chemotherapy. Cancer 2016; 122: 2646-2653. 
11. Hadji P, Body JJ, Aapro MS et al. Practical guidance for the management of aromatase inhibitorassociated bone loss. Ann Oncol 2008; 19: 1407-1416.

12. Chlebowski RT, Aragaki AK, Anderson GL et al. Dietary Modification and Breast Cancer Mortality: Long-Term Follow-Up of the Women's Health Initiative Randomized Trial. J Clin Oncol 2020; JC01900435.

13. Limon-Miro AT, Lopez-Teros V, Astiazaran-Garcia H. Dietary Guidelines for Breast Cancer Patients: A Critical Review. Adv Nutr 2017; 8: 613-623.

14. Runowicz CD, Leach CR, Henry NL et al. American Cancer Society/American Society of Clinical Oncology Breast Cancer Survivorship Care Guideline. CA Cancer J Clin 2016; 66: 43-73.

15. Martinez-Gonzalez MA, Fernandez-Jarne E, Serrano-Martinez M et al. Development of a short dietary intake questionnaire for the quantitative estimation of adherence to a cardioprotective Mediterranean diet. Eur J Clin Nutr 2004; 58: 1550-1552.

16. Weigl J, Hauner H, Hauner D. Can Nutrition Lower the Risk of Recurrence in Breast Cancer? Breast Care (Basel) 2018; 13: 86-91.

17. Ligibel JA, Basen-Engquist K, Bea JW. Weight Management and Physical Activity for Breast Cancer Prevention and Control. Am Soc Clin Oncol Educ Book 2019; 39: e22-e33.

18. Reeves MM, Terranova CO, Eakin EG, Demark-Wahnefried W. Weight loss intervention trials in women with breast cancer: a systematic review. Obes Rev 2014; 15: 749-768.

19. Janni W, Rack B, Friedl T et al. Abstract GS5-03: Lifestyle Intervention and Effect on Disease-free Survival in Early Breast Cancer Pts: Interim Analysis from the Randomized SUCCESS C Study. Cancer Research 2019; 79: GS5-03-GS05-03.

20. Trestini I, Carbognin L, Monteverdi S et al. Clinical implication of changes in body composition and weight in patients with early-stage and metastatic breast cancer. Crit Rev Oncol Hematol 2018; 129 : 54-66.

21. Caan BJ, Emond JA, Natarajan $L$ et al. Post-diagnosis weight gain and breast cancer recurrence in women with early stage breast cancer. Breast Cancer Res Treat 2006; 99: 47-57.

22. Trestini I, Carbognin L, Bonaiuto $C$ et al. The obesity paradox in cancer: clinical insights and perspectives. Eat Weight Disord 2018; 23: 185-193.

23. Chlebowski RT, Aiello E, McTiernan A. Weight loss in breast cancer patient management. J Clin Oncol 2002; 20: 1128-1143.

24. Gal R, Monninkhof EM, Peeters PHM et al. Physical activity levels of women with breast cancer during and after treatment, a comparison with the Dutch female population. Acta Oncol 2019; 58 : 673-681.

25. Rock CL, Doyle C, Demark-Wahnefried W et al. Nutrition and physical activity guidelines for cancer survivors. CA Cancer J Clin 2012; 62: 243-274.

26. Vance V, Mourtzakis M, McCargar L, Hanning R. Weight gain in breast cancer survivors: prevalence, pattern and health consequences. Obes Rev 2011; 12: 282-294. 
27. Chlebowski RT, Aragaki AK, Anderson GL et al. Association of Low-Fat Dietary Pattern With Breast Cancer Overall Survival: A Secondary Analysis of the Women's Health Initiative Randomized Clinical Trial. JAMA Oncol 2018; 4: e181212.

28. Maskarinec G, Morimoto $Y$, Takata $Y$ et al. Alcohol and dietary fibre intakes affect circulating sex hormones among premenopausal women. Public Health Nutr 2006; 9: 875-881.

29. Wu AH, Pike MC, Stram DO. Meta-analysis: dietary fat intake, serum estrogen levels, and the risk of breast cancer. J Natl Cancer Inst 1999; 91: 529-534.

30. Kwok A, Palermo C, Boltong A. Dietary experiences and support needs of women who gain weight following chemotherapy for breast cancer. Support Care Cancer 2015; 23: 1561-1568.

31. de Liz S, Vieira FGK, de Assis MAA et al. Adherence to the WCRF/AICR for Women in Breast Cancer Adjuvant Treatment submitted to Educational Nutritional Intervention. Nutr Cancer 2018; 70: 737747.

32. Thomson ZO, Reeves MM. Can weight gain be prevented in women receiving treatment for breast cancer? A systematic review of intervention studies. Obes Rev 2017; 18: 1364-1373.

33. Chlebowski RT, Reeves MM. Weight Loss Randomized Intervention Trials in Female Cancer Survivors. J Clin Oncol 2016; 34: 4238-4248.

34. Kirkham AA, Van Patten CL, Gelmon KA et al. Effectiveness of Oncologist-Referred Exercise and Healthy Eating Programming as a Part of Supportive Adjuvant Care for Early Breast Cancer. Oncologist 2018; 23: 105-115.

35. Djuric Z, DiLaura NM, Jenkins I et al. Combining weight-loss counseling with the weight watchers plan for obese breast cancer survivors. Obes Res 2002; 10: 657-665.

36. Djuric Z, Mirasolo J, Kimbrough L et al. A pilot trial of spirituality counseling for weight loss maintenance in African American breast cancer survivors. J Natl Med Assoc 2009; 101: 552-564.

37. Salas-Salvado J, Diaz-Lopez A, Ruiz-Canela M et al. Effect of a Lifestyle Intervention Program With Energy-Restricted Mediterranean Diet and Exercise on Weight Loss and Cardiovascular Risk Factors: One-Year Results of the PREDIMED-Plus Trial. Diabetes Care 2019; 42: 777-788.

38. Shaikh AA, Braakhuis AJ, Bishop KS. The Mediterranean Diet and Breast Cancer: A Personalised Approach. Healthcare (Basel) 2019; 7.

39. Pierce JP, Natarajan L, Caan BJ et al. Influence of a diet very high in vegetables, fruit, and fiber and low in fat on prognosis following treatment for breast cancer: the Women's Healthy Eating and Living (WHEL) randomized trial. JAMA 2007; 298: 289-298.

40. Finocchiaro $\mathrm{C}$, Ossola M, Monge $T$ et al. Effect of specific educational program on dietary change and weight loss in breast-cancer survivors. Clin Nutr 2016; 35: 864-870.

41. Villarini $A$, Pasanisi $P$, Raimondi $M$ et al. Preventing weight gain during adjuvant chemotherapy for breast cancer: a dietary intervention study. Breast Cancer Res Treat 2012; 135: 581-589.

42. Zuniga KE, Parma DL, Munoz E et al. Dietary intervention among breast cancer survivors increased adherence to a Mediterranean-style, anti-inflammatory dietary pattern: the Rx for Better Breast Health 
Randomized Controlled Trial. Breast Cancer Res Treat 2019; 173: 145-154.

43. Moon HG, Han W, Noh DY. Underweight and breast cancer recurrence and death: a report from the Korean Breast Cancer Society. J Clin Oncol 2009; 27: 5899-5905.

44. Marret $H$, Perrotin F, Bougnoux $P$ et al. Low body mass index is an independent predictive factor of local recurrence after conservative treatment for breast cancer. Breast Cancer Res Treat 2001; 66: 1723.

45. Arpino G, De Angelis $C$, Buono $G$ et al. Metabolic and anthropometric changes in early breast cancer patients receiving adjuvant therapy. Breast Cancer Res Treat 2015; 154: 127-132.

46. Berrino F, Villarini A, Traina A et al. Metabolic syndrome and breast cancer prognosis. Breast Cancer Res Treat 2014; 147: 159-165.

47. van den Berg MM, Winkels RM, de Kruif JT et al. Weight change during chemotherapy in breast cancer patients: a meta-analysis. BMC Cancer 2017; 17: 259.

48. Pila E, Sabiston CM, Taylor VH, Arbour-Nicitopoulos K. "The Weight Is Even Worse Than the Cancer": Exploring Weight Preoccupation in Women Treated for Breast Cancer. Qual Health Res 2018; 28 : 1354-1365.

49. Bosy-Westphal A, Booke CA, Blocker T et al. Measurement site for waist circumference affects its accuracy as an index of visceral and abdominal subcutaneous fat in a Caucasian population. $J$ Nutr 2010; 140: 954-961.

50. Villarini A, Pasanisi P, Traina A et al. Lifestyle and breast cancer recurrences: the DIANA-5 trial. Tumori 2012; 98: 1-18.

51. Ligibel JA, Barry WT, Alfano $C$ et al. Randomized phase III trial evaluating the role of weight loss in adjuvant treatment of overweight and obese women with early breast cancer (Alliance A011401): study design. NPJ Breast Cancer 2017; 3: 37.

\section{Tables}

Table 1. Baseline patients' characteristics. 
Median age in years (range)

Median follow-up in months (range)

Menopausal state at diagnosis

Premenopausal

Postmenopausal

pT

0

1

2

3

4

Unknown

$\mathrm{pN}$

0

1-3

Unknown

Histological Grade of primary

1

2

3

Unknown

Ki67 Status

$<20 \%$

$>20 \%$

Unknown

HER2 Status

Positive
49 (27-83)

24 (1-157)

131 (53.9\%)

$112(46.0 \%)$

24 (9.9\%)

132 (54.3\%)

68 (28.0\%)

8 (3.3\%)

$2(0.8 \%)$

$9(3.7 \%)$

121 (49.8\%)

119 (49.0\%)

$3(1.2 \%)$

20 (8.2\%)

95 (39.1\%)

93 (38.3\%)

35 (14.4\%)

107 (44.0\%)

134 (55.2\%)

$2(0.8 \%)$

$80(32.9 \%)$ 
Negative

Unknown

Estrogen Receptor Status

Positive

Negative

Unknown

Progesteron Receptor Status

Positive

Negative

Unknown

Breast cancer phenotype

Luminal $A$

Luminal B

HER2-positive/luminal

HER2-positive non luminal

Triple Negative

Not evaluable

Axillary dissection

Yes

No

Unknown

Radiation treatment

Yes

No

Adjuvant chemotherapy

Yes

No

Adjuvant hormonotherapy

Yes

No
$162(66.7 \%)$

$1(0.4 \%)$

$190(78.2 \%)$

$33(13.6 \%)$

$20(8.2 \%)$

153 (63.0)

64 (26.3)

26 (10.7)

$84(34.6 \%)$

$59(24.3 \%)$

$61(25.1 \%)$

$19(7.8 \%)$

$18(7.4 \%)$

$2(0.8 \%)$

$112(46.1 \%)$

$128(52.7 \%)$

$3(1.2 \%)$

156 (64.2\%)

$87(35.8 \%)$

118 (48.6\%)

125 (51.4\%)

202 (83.1\%)

41 (16.9\%) 


\section{Adjuvant Trastuzumab}

Yes

$51(21.0 \%)$

No

$192(79.0 \%)$

Neoadjuvant chemotherapy

Yes

$67(27.6 \%)$

No

$176(72.4 \%)$

Pathologic Complete Response to NACT (of those who underwent NACT, $\mathrm{N}=67$ )

Yes

$22(32.8 \%)$

No

$42(62.7 \%)$

Not available

$3(0.4 \%)$

Neoadjuvant Trastuzumab

Yes

$27(11.1 \%)$

No

$216(88.9 \%)$

Median fasting glycemia $(\mathrm{mg} / \mathrm{dl})$ [IQR]

91 (85.5-99.0)

Median triglycerides (mg/dl) [IQR]

$93(68.5-134.5)$

Median total cholesterol (mg/dl) [IQR]

200.5 (171.8-

223.0)

Median HDL cholesterol (mg/dl) [IQR]

$62(52.0-70.0)$

Physical activity level

Inactive

$191(79 \%)$

Minimally active

$52(21 \%)$

Sufficiently active

$0(0 \%)$

Legend - Table 1: NACT, Neoadjuvant Chemotherapy; IQR, interquartile range.

Table 2. Baseline nutritional features in the overall population and according to menopausal status. 


\begin{tabular}{|c|c|c|c|c|}
\hline Variable & $\begin{array}{l}\text { All } \\
(234 \\
\text { Patients) }\end{array}$ & $\begin{array}{l}\text { Premenopausal } \\
\text { status } \\
\text { (131 Patients) }\end{array}$ & $\begin{array}{l}\text { Postmenopausal } \\
\text { status } \\
\text { (112 Patients) }\end{array}$ & $\begin{array}{l}p- \\
\text { value }\end{array}$ \\
\hline $\begin{array}{l}\text { Median usual body weight } \\
\text { (Kg) [IQR] }\end{array}$ & $\begin{array}{l}63(57.0- \\
71.3)\end{array}$ & $64.0(57.0-72.0)$ & $62.4(57.0-70.0)$ & $0.71^{*}$ \\
\hline Median body weight (Kg) [IQR] & $\begin{array}{l}67.4(58.5- \\
76.0)\end{array}$ & $67.2(58.7-75.8)$ & $67.4(59.0-76.9)$ & $0.66^{*}$ \\
\hline Median BMI $\left(\mathrm{Kg} / \mathrm{m}^{2}\right)$ [IQR] & $\begin{array}{l}25.6(22.5- \\
29.1)\end{array}$ & $25.5(22.0-29.0)$ & $25.6(23.1-29.3)$ & \multirow[t]{5}{*}{$0.56^{*}$} \\
\hline Underweight N. (\%) & $5(2.1 \%)$ & $5(3.8 \%)$ & 0 & \\
\hline Normal weight N. (\%) & $87(35.8 \%)$ & $50(20.6 \%)$ & $37(33.0 \%)$ & \\
\hline Overweight N. (\%) & $93(38.2 \%)$ & $46(35.1 \%)$ & $47(42.0 \%)$ & \\
\hline Obesity N. (\%) & $58(23.9 \%)$ & $30(22.9 \%)$ & $28(25.0 \%)$ & \\
\hline Weight gain $\geq 5 \%$ N. (\%) & $115(47.3 \%)$ & $61(46.6 \%)$ & $54(48.2 \%)$ & $0.80^{\#}$ \\
\hline Median WC (cm) [IQR] & $\begin{array}{l}86(77.0- \\
95.0)\end{array}$ & $83.5(75.0-94.0)$ & $88(81.0-97.0)$ & $0.01 *$ \\
\hline Central obesity N. (\%) & $128(52.7 \%)$ & $59(44.0 \%)$ & $69(63.3 \%)$ & $0.01^{\#}$ \\
\hline
\end{tabular}

Legend - Table 2: IQR, interquartile range; BMI, Body Mass Index; WC, Waist Circumference; * $p$-value: Mann-Whitney non parametric test; ${ }^{\#} p$-value: Pearson's chi-square test.

Table 3. Predictors of BMI change at univariate and multivariate analysis. 


\begin{tabular}{|c|c|c|c|c|c|c|}
\hline \multirow[t]{2}{*}{ Variables } & \multicolumn{3}{|c|}{ Univariate Analysis } & \multicolumn{3}{|c|}{ Multivariate Analysis } \\
\hline & ORs & $\begin{array}{l}95 \% \\
\mathrm{Cl}\end{array}$ & $\begin{array}{l}p- \\
\text { value }\end{array}$ & ORs & $\begin{array}{l}95 \% \\
\mathrm{Cl}\end{array}$ & $\begin{array}{l}p \text { - } \\
\text { value }\end{array}$ \\
\hline $\begin{array}{l}\text { Age at diagnosis } \\
\text { (<70 years } v s \geq 70 \text { years) }\end{array}$ & 1.86 & $\begin{array}{l}0.47- \\
7.38\end{array}$ & 0.376 & & & \\
\hline $\begin{array}{l}\text { Menopausal status } \\
\text { (Premenopausal vs postmenopausal) }\end{array}$ & 1.93 & $\begin{array}{l}1.15- \\
3.25\end{array}$ & 0.013 & 2.09 & $\begin{array}{l}1.23- \\
3.55\end{array}$ & 0.007 \\
\hline $\begin{array}{l}\text { Lymph Node Status } \\
\text { (Negative vs positive) }\end{array}$ & 1.54 & $\begin{array}{l}0.92- \\
2.57\end{array}$ & 0.102 & & & \\
\hline $\begin{array}{l}\text { Treatment } \\
\text { (chemotherapy vs chemotherapy+ } \\
\text { hormonotherapy vs hormonotherapy) }\end{array}$ & 0.82 & $\begin{array}{l}0.45- \\
1.52\end{array}$ & 0.53 & & & \\
\hline $\begin{array}{l}\text { Adherence to dietary guidelines } \\
\text { (High vs low) }\end{array}$ & 4.62 & $\begin{array}{l}1.24- \\
17.3\end{array}$ & 0.023 & 5.46 & $\begin{array}{l}1.43- \\
20.85\end{array}$ & 0.013 \\
\hline
\end{tabular}

Legend - Table 3: ORs, Odds ratios; $\mathrm{Cl}$, confidence interval; vs, versus.

\section{Figures}



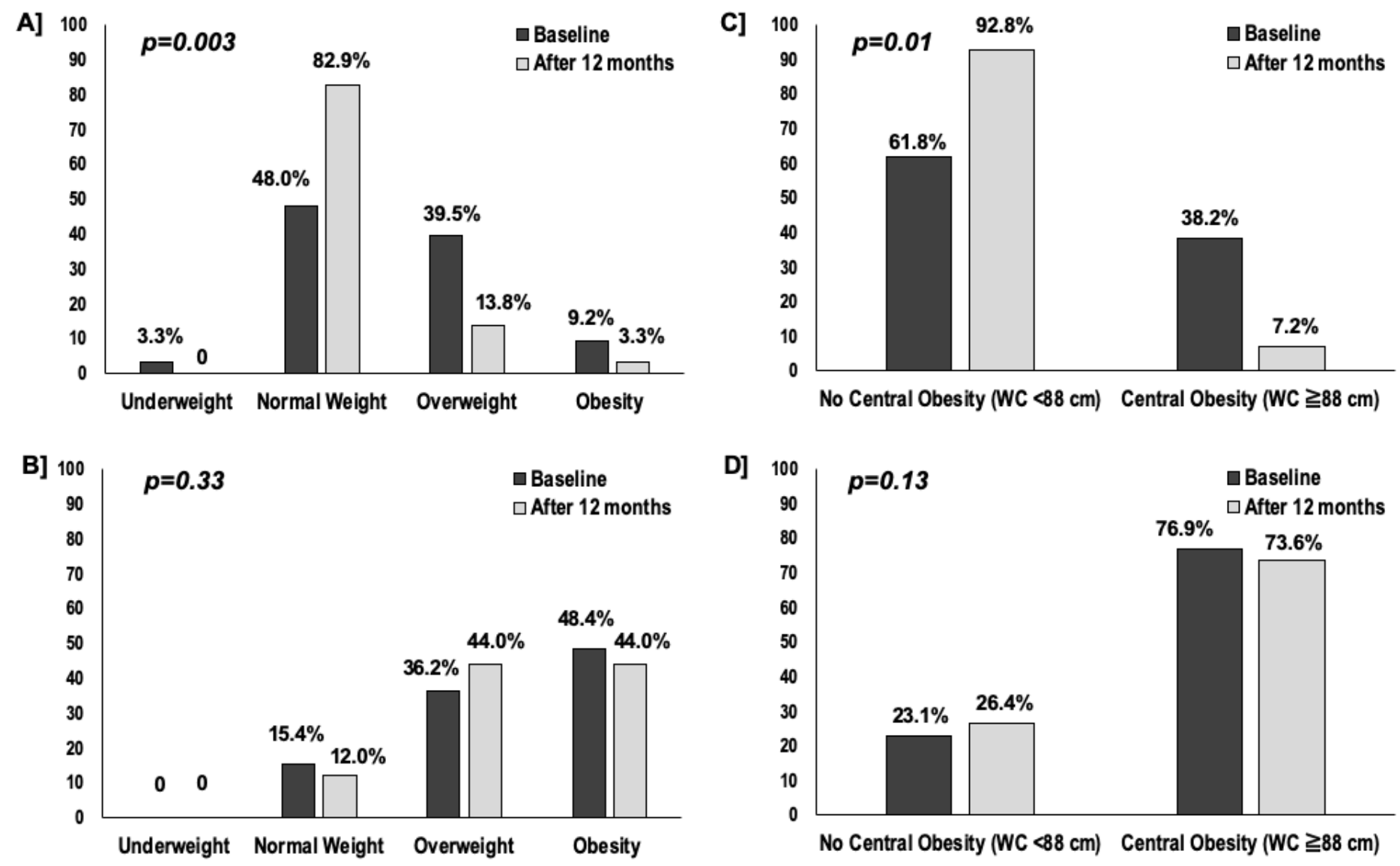

\section{Figure 1}

Body Mass Index (classification according to World Health Organization criteria) and waist circumference change after the dietary intervention in patients with a high adherence [Panel A-C] and low adherence [Panel B-D] to dietary guidelines. WC, Waist Circumference; p-value: Mc-Nemar non parametric paired test.
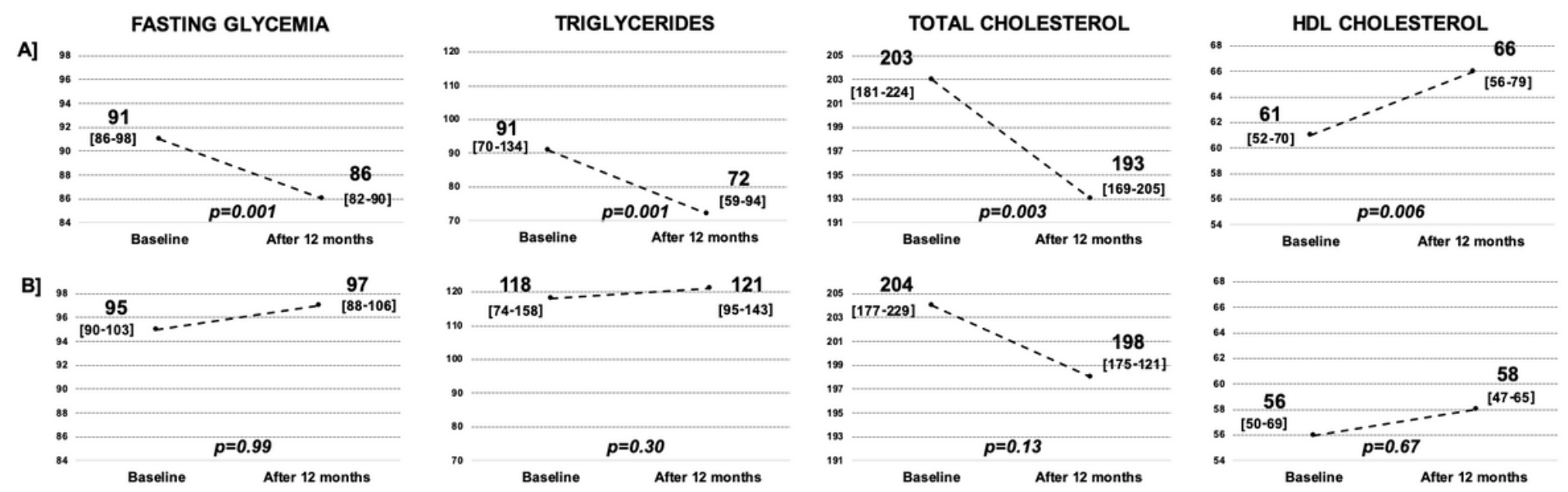

Figure 2

Blood glucose and lipid profile tests' change after the dietary intervention in patients with a high adherence [Panel A] and low adherence [Panel B] to dietary guidelines. HDL, high-density lipoprotein; 
fasting glycemia, triglycerides, total cholesterol and HDL cholesterol values are measured in $\mathrm{mg} / \mathrm{dl}$; Interquartile Range are reported in square brackets; p-value: Wilcoxon non-parametric paired test.

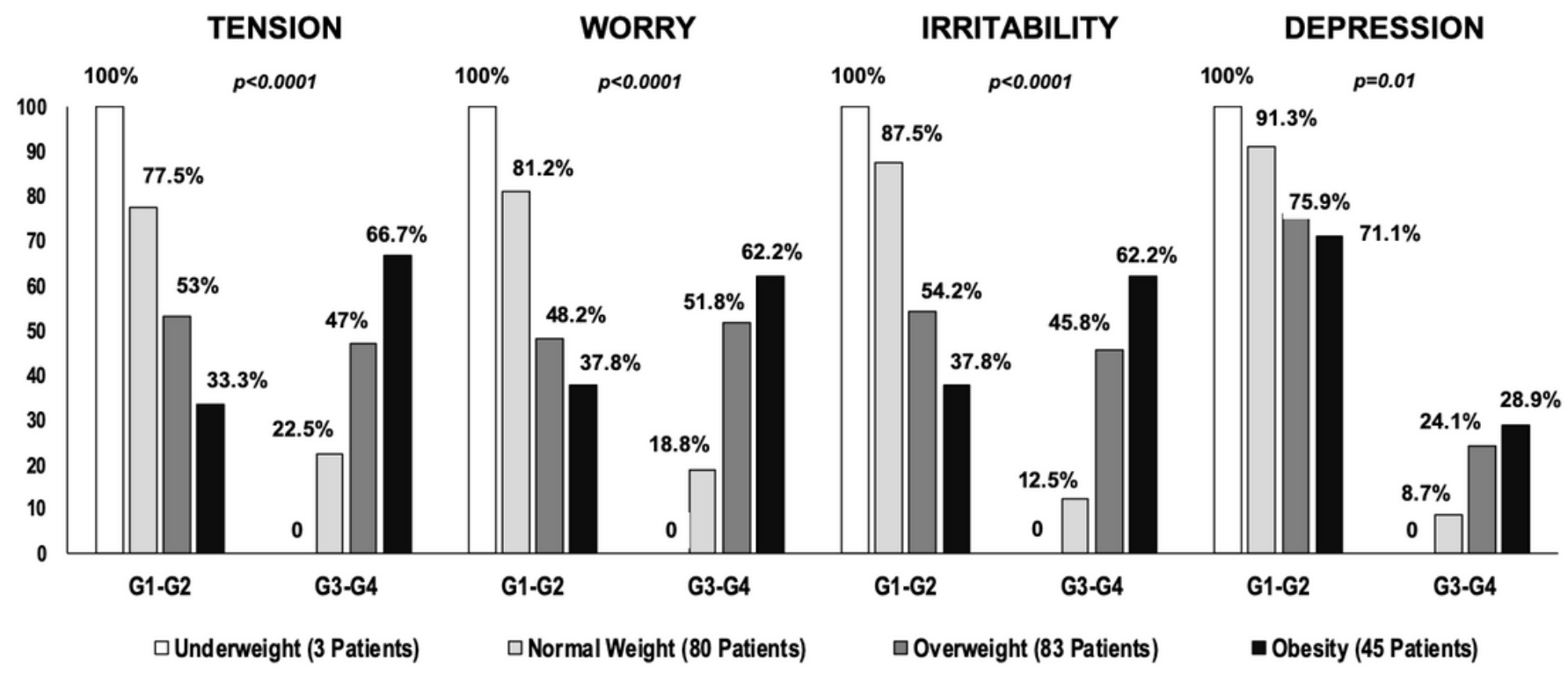

Figure 3

Relationship between emotional function and baseline Body Mass Index (classification according to World Health Organization criteria). p-value: Pearson's chi-square test.

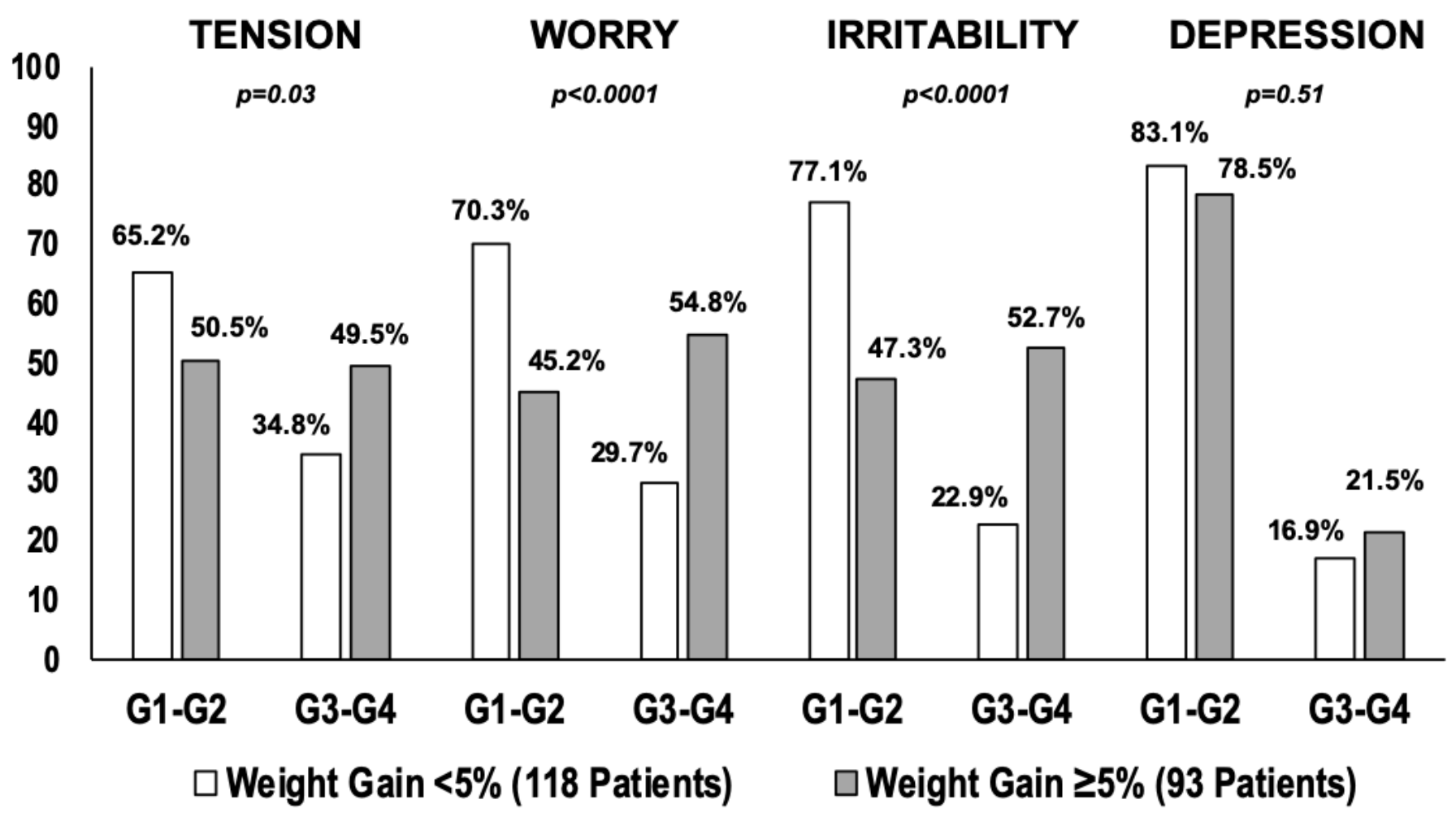

Figure 4 
Associations between emotional function and weight gain at baseline. p-value: Pearson's chi-square test. 